\title{
ESTRATEGIAS PARA UNA COMUNIDAD SALU- DABLE: VALORACIÓN DEL ESTADO NUTRICIONAL DE UN MUESTREO POBLACIONAL A TRAVÉS DE MEDICIONES ANTROPOMÉTRICAS
}

Bqco. Eduardo Ariel Leyes Pedrozo - Bqca. Silvina María Martínez - Esp. Lucrecia Ghione - Esp. Natalia Andrea Serrano - Esp. Patricia Noemí Goicoechea - Esp. Ma. del Carmen Gauna Pereira $\left(^{*}\right)$

Palabras Claves: Nutrición - Parámetros pondoestaturales - Salud

\section{Resumen}

En el marco del Programa de Mejora Barrial (PROMEBA), se encuestaron y obtuvieron muestras de sangre venosa a 190 personas de ambos sexos y edades comprendidas entre 1 y 70 años, con el fin de generar conocimiento preliminar acerca del estado nutricional de un grupo muestral perteneciente a un complejo barrial de unas 9325 personas ubicado al sudeste de la ciudad Capital de la Provincia de Corrientes. La actividad económica desarrollada por los habitantes es temporaria y ocasional, contando con escasa asistencia en salud y en un contexto barrial deficiente en las condiciones mínimas de vida.

Se trabajó con los datos pondoestaturales medidos al grupo muestra, para estimar el estado nutricional a priori de las mediciones bioquímicas a ser efectuadas. Se trabajó con balanza médica mecánica para adultos con Tallímetro, para la obtención del peso y la talla y luego se calculó el Índice de Masa Corporal (IMC), utilizando Microsoft Excel y Minitab 15 y compa- rando dos métodos de clasificación con el objeto de establecer si se puede cualificar el estado nutricional de un individuo usando uno $\mathrm{u}$ otro método independientemente (aplicación de criterios en el proceso de enseñanza - aprendizaje de los alumnos). Salvo excepciones, los valores de peso y talla se correspondían con los calculados para el IMC pero tanto en población joven como en adulta, existe un porcentaje variable al que se debería hacer un perfil bioquímico nutricional en todos los casos y finalmente un estudio clínico para concluir con un diagnóstico y tratamiento adecuado.

\section{Summary}

Under the Neighborhood Improvement Program (PROMEBA), were surveyed, and 190 blood samples were obtained of both sexes and aged between 1 and 70 years, in order to generate preliminary knowledge about the nutritional status of a sample group belonging to a settlement of some 9325 residents of a neighborhood complex located southeast of the capital city of the Province of Corrientes. The activity carried out by them is temporary and occasional, with low economic resources, poor assistance in health and poor neighborhood context in the minimum living conditions. 
We worked with the information pondoestaturales measured to the group, To estimate the nutritional state of the a priori biochemical measurements to be made. We worked with a mechanical medical scales for adults with height rod for getting weight and height and then the Body Mass Index (BMI) was calculated using Microsoft Excel and Minitab 15 and comparing two classification methods in order to establish whether can qualify the nutritional status of an individual using one or another method independently (applying criteria in the process of teaching - learning of the students). With few exceptions, the values of weight and height were consistent with those calculated for BMI but both young and adult population, there is a variable percentage that should make a nutritional biochemical profile in all cases and finally a clinical study to conclude with a diagnosis and appropriate treatment.

\section{INTRODUCCIÓN}

Dada la importancia de la salud en el bienestar directo de las personas, se han establecido organizaciones de distinto nivel de alcance para monitorear e identificar los posibles factores que impacten en la salud, como el estado de la población objeto de estudio, a modo de establecer normas de profilaxis, regulación de los medicamentos e insumos utilizados y establecer normas de tratamiento de enfermedades. Además, existe consenso mundial que la salud es un bien público y necesario para el bienestar de la sociedad y por tal motivo los estados, sin excepción, toman parte en el tema.

Es importante agregar que, antes de proponer un proyecto determinado, es fundamental efectuar un diagnóstico de situación en la zona donde se percibe la existencia de un problema o necesidad relacionada con la salud de las personas. El principal objetivo del proyecto, es conocer dicho estado de salud, hábitat y principales actividades que realizan quienes residen es la zona de interés.
En este contexto, se buscó estimar el estado nutricional de un grupo muestra, a través de los datos pondoestaturales medidos a dicho grupo, previo a las mediciones bioquímicas a ser efectuadas. Estos individuos pertenecen a un asentamiento de unos 9325 habitantes de un complejo barrial ubicado al sudeste de la ciudad Capital de la Provincia de Corrientes. La actividad económica desarrollada por los habitantes es temporaria y ocasional, contando con escasa asistencia en salud y en un contexto barrial deficiente en las condiciones mínimas de vida.

\section{MATERIALES Y MÉTODOS}

Muestra: Individuos clínicamente sanos, adultos y niños de ambos sexos.

Número total de personas encuestadas: 190 - Adultos: 90 (Hombres: 16-Mujeres: 74) - Niños y adolescentes: 100 (Femeninos: 56 - Masculinos:44)

Materiales: Balanza médica mecánica para adultos con Tallímetro

Softwares presentación: Microsoft Word - Power Point - Jasc Paint Shop Pro 9

Softwares estadística: Microsoft Excel Minitab 15 (Español)

Método: La población se dividió en dos grandes grupos:

- Grupo I: adultos con edades mayores de 20 años.

- Grupo II: niños y adolescentes con edades de 1 a 20 años

A ambos grupos se les determinó:

- Peso y Talla

- IMC (Índice de Quetelet o indice de Masa Corporal)

$$
\mathrm{IMC}=\frac{\text { masa }}{\text { estatura }^{2}}
$$

- \% PI = Peso Real x 100/ Peso Ideal 


\section{RESULTADOS}

Según sexo y edad - Peso vs Edad:

\section{Gráfico No 1}
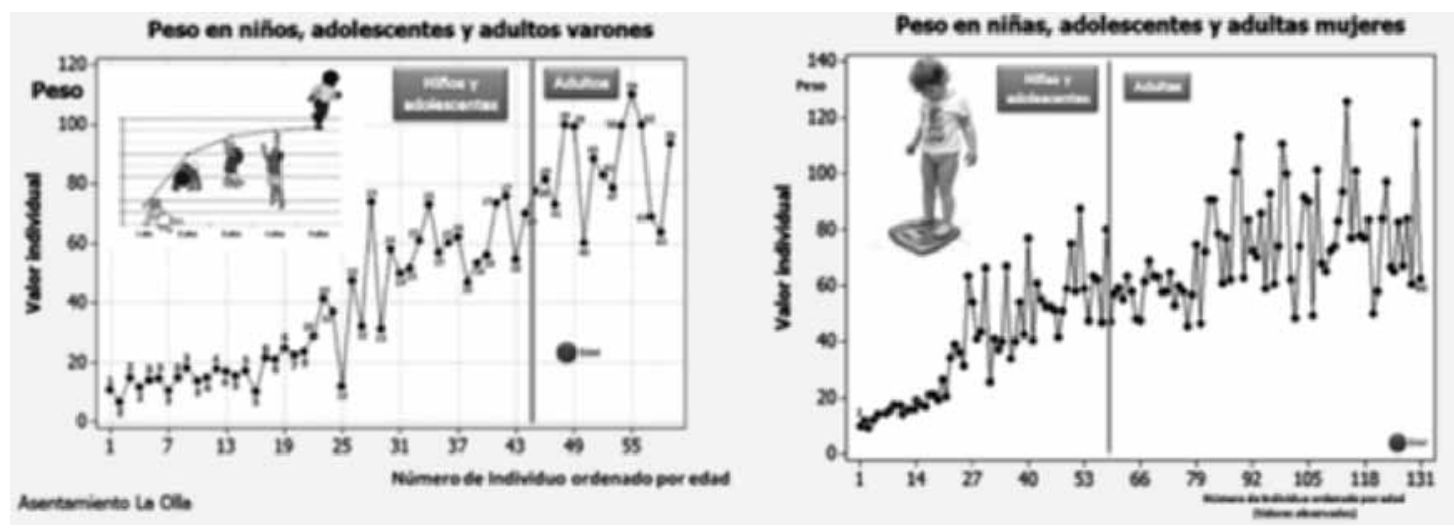

Según sexo y edad - Talla vs Edad: Gráfico No 2
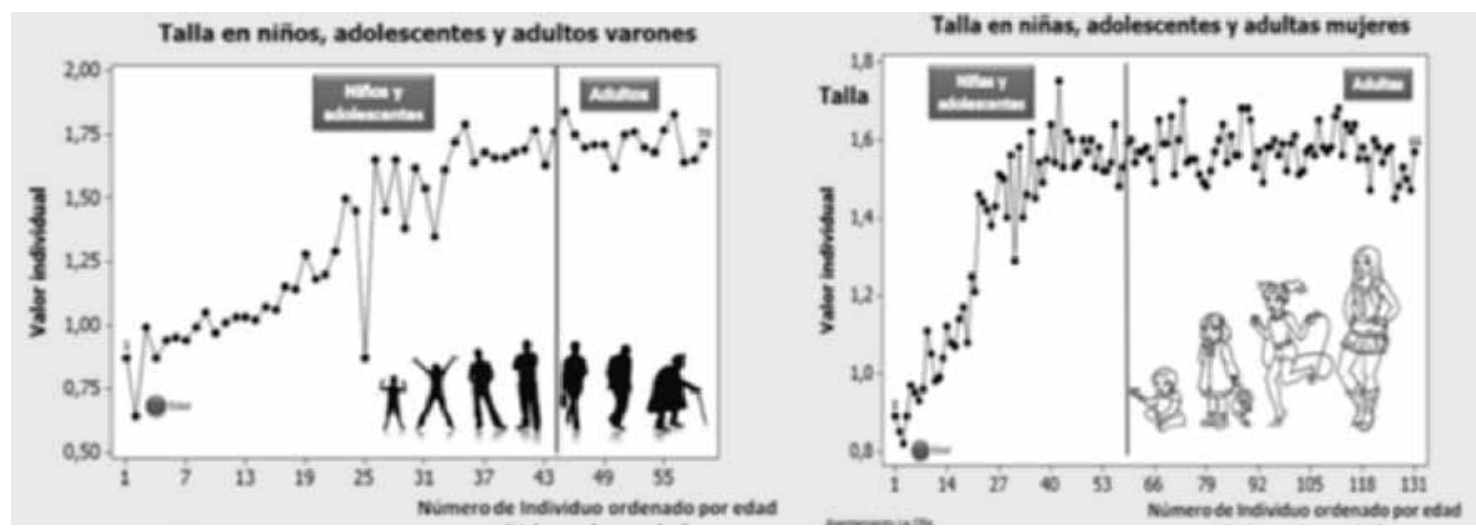

Análisis de los resultados en niños, niñas y adolescentes de ambos sexos Talla:

Percentiles o percentilos para Peso y

En el grupo de niñas y mujeres adolescentes estudiado $(n=56)$, el $5,26 \%$ se encuenta por debajo de la línea $Z=-2$ en peso (Percentilo 3) y talla (Percentilo 5). Si bien no es necesario una correlación equivalente de percentilos entre peso y talla, el 7\% presenta una diferencia muy amplia en dichos valores, con una inclinación a favor por el percentilo del peso en relación con el percentilo de la talla, sin hacer una clasificación entre sobrepeso y obesidad.
En el grupo de niños y varones adolescentes $(n=44)$, estas mismas cifras representan el $6,82 \%$ por debajo $Z=-2$, con sólo un $2.27 \%$ de marcada diferencia entre ambos percentilos.

Los restantes casos en ambas poblaciones, se ajustan dentro de los valores de referencia para peso y talla en este grupo etáreo en ambos sexos (1 a 19 años).

Con respecto al IMC (Índice de Quetelet), en coincidencia con publicaciones consultadas, esta expresión matemática no se ajusta con lo observado para peso y talla en el grupo de niñas, niños y adolescentes de ambos sexos. Aplicando percentilos, en va- 
rones se observan tres casos con valores por debajo de $Z=-2$ (Percentilo 5) y dos casos por encima de $Z=+2$ (Percentilo 95) y en mujeres se observa un solo caso de valor por debajo de $Z=-2$ (menor a Percentilo 5) y cinco casos de valores por encima de $Z=+2$ (Percentilo 95). En todos los casos hubo correspondencia con los respectivos hallazgos en pesos y tallas.

\section{En adultos de ambos sexos}

Cálculo del IMC y comparación con fórmulas ponderadas
Se analizaron los datos aplicando la relación entre el peso ideal (obtenido por una expresión algebraica ponderada) y se estableció la relación con el peso real (\% PI). Al mismo tiempo se calculó el IMC (Índice de Quetelet) y sus clasificaciones respectivas (expresadas en porcentajes) fueron comparados por el Método de Fleiss (Medida de concordancia para variables cualitativas: Índice Kappa), con el objeto de establecer si se puede cualificar el estado nutricional de un individuo usando uno u otro método independientemente.

Los resultados calificados por ambos métodos se exponen en la Gráfica No 5 y Gráfica No 6 .

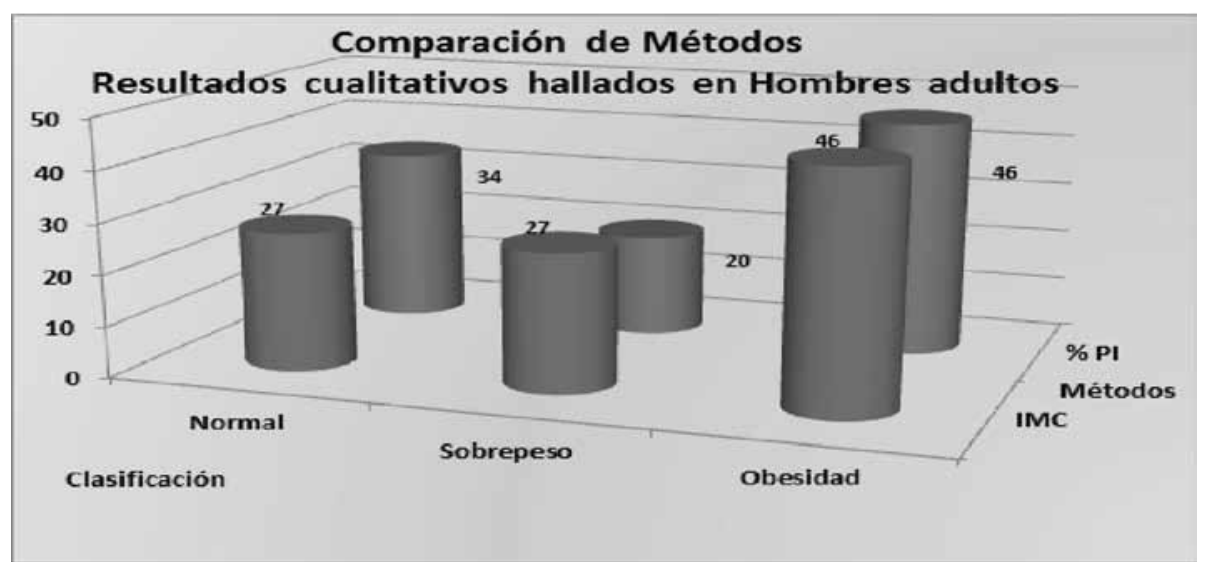

Gráfica No 5 - IMC y \% PI en Hombres adultos

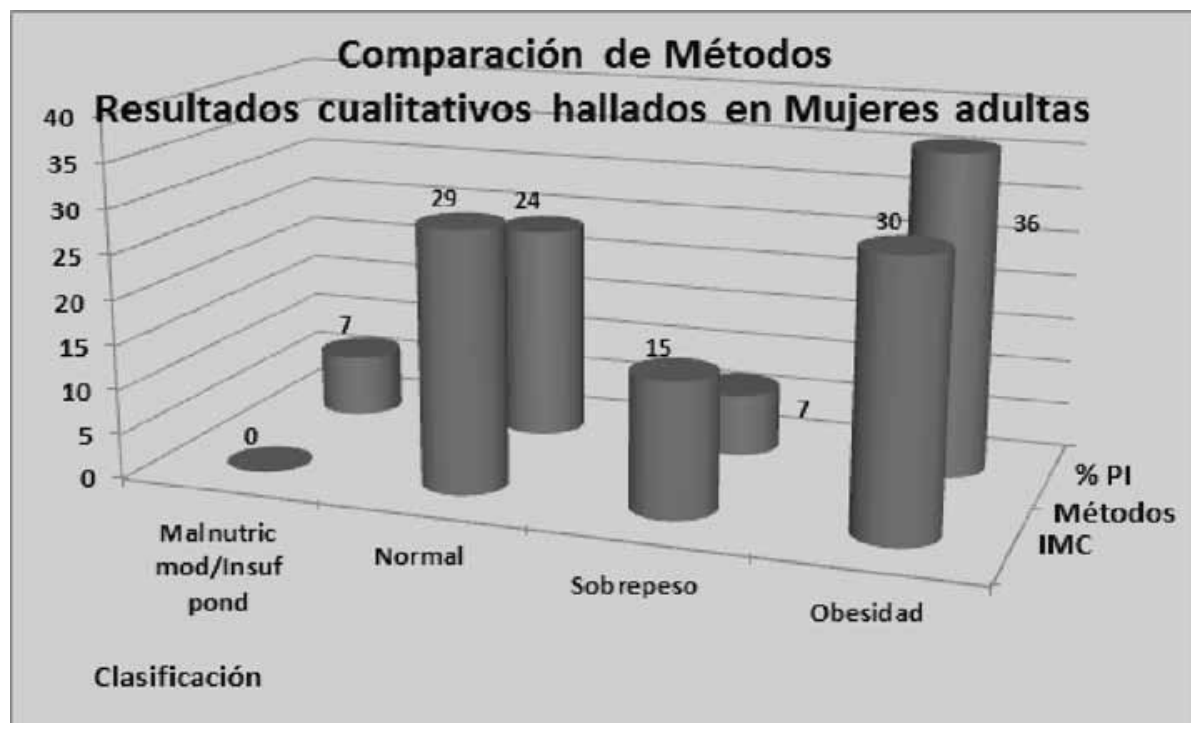

Gráfica No 6 - IMC y \% PI en Mujeres adultas 
En ambos métodos, no son despreciables los porcentajes correspondientes a las categorías sobrepeso y obeso (IMC: $73 \%$ \% PI: 66\%) en varones y (IMC: 45\% - \% PI: 43\%) en mujeres. En el otro extremo, no se registran deficiencias ponderales en hombres por ambos métodos, mientras que en mujeres sólo califica el método de \% PI con un $7 \%$.

\section{Conclusiones preliminares}

Los resultados llevan a establecer cate- gorías que, seguidamente, deben ser evaluadas a través de un perfil bioquímico nutricional en todos los casos y finalmente un estudio clínico para concluir con un diagnóstico adecuado.

Por otra parte, el número de la muestra tiene mucha importancia para aplicar su utilidad al estudio de grandes poblaciones, sin descuidar los casos particulares que necesitan ser llevados al área clínica para su tratamiento y seguimiento.

\section{BIBLIOGRAFÍA}

- Rodríguez Martínez, G.; Moreno Aznar, L.; Sarría Chueca, A.: Sobre el índice de Quetelet y Obesidad. Revista Española de Obesidad. Vol 8. No 1: 34 - 40. 2010.

- Mezquita Pla, Cristóbal y colaboradores: Fisiología Médica: del razonamiento fisiológico al razonamiento clínico. Editorial Médica Panamericana. Madrid. 2011.

- Dvorkin, M.A.; Cardinali, D.P.; Iermoli, R.H.: Best \& Taylor. Bases Fisiológicas de la Práctica Médica. 14a Edición. Editorial médica Panamericana. Buenos Aires. 2010.

- www.seedo.es/LinkClick.aspx?...Obesidadhistoria-Sobre-2

- http://www.who.int/childgrowth/es/

- http://www.nutricion.org/publicaciones/ pdf/antropometria.pdf

- http://www.elizalde.gov.ar/area_medica/ ateneos/tablas $\% 20 \mathrm{de} \% 20$ crecimiento $\% 20$ ferraro.pdf1

- http://www.msc.es/biblioPublic/publicaciones/recursos_propios/resp/revista_cdrom/ VOL66/66_1_065.pdf

- http://www.fmed.uba.ar/depto/nutrievaluacion/GRAFICOS\%20Y\%20TABLAS\%20 TP\%201\%20a\%207.pdf

- http://www.seh-lelha.org/pdf/concor2.pdf
- http://aborges.webs.ull.es/coeficiente $\% 20$ kappa.pdf

- http://www.fisterra.com/mbe/investiga/kappa/kappa2.pdf

- http://www.sergas.es/gal/documentacionTecnica/docs/SaudePublica/Apli/Epidat4/ Ayuda/Concordancia\%20y\%20consistencia.pdf

- http://lbe.uab.es/vm/sp/materiales/bloque-2/habitos/Capitulo0203.pdf

- http://www.bebesymas.com/salud-infantil/ obesidad-infantil-como-calcular-el-imcde-los-ninos-y-valorarlo-en-las-tablas

- ww.facmed.unam.mx/deptos/salud/censenanza/spi/unidad2/Antropometria_manualinnsz.pdf

- http://www.bvs.ins.gob.pe/insprint/cenan/ modulo_medidas_antropometricas_registro_estandarizacion.pdf

- http://www.who.int/childgrowth/standards/chts_bfa_ninos_p/es/

Tabla en niñas

www.cdc.gov/growthcharts/data/.../co061023. pdf

Tabla en niños

www.cdc.gov/growthcharts/data/.../co061024. pdf 